\title{
ARTICLE
}

Clinical Research

\section{The impact of obesity on COVID-19 complications: a retrospective cohort study}

\author{
Mohamed Nakeshbandi $\mathbb{D}^{1} \cdot$ Rohan Maini $\mathbb{D}^{2} \cdot$ Pia Daniel $^{2} \cdot$ Sabrina Rosengarten $\mathbb{D}^{2} \cdot$ Priyanka Parmar $\mathbb{B}^{2} \cdot$ \\ Clara Wilson $\mathbb{D}^{2} \cdot$ Julie Minjae $\operatorname{Kim} \mathbb{D}^{2} \cdot$ Alvin Oommen $\mathbb{D}^{2} \cdot \operatorname{Max}$ Mecklenburg $\mathbb{D}^{2} \cdot$ Jerome Salvani $\mathbb{D}^{3} \cdot$ \\ Michael A. Joseph ${ }^{4} \cdot$ Igal Breitman ${ }^{5}$
}

Received: 23 May 2020 / Revised: 24 June 2020 / Accepted: 16 July 2020 / Published online: 25 July 2020

(C) The Author(s), under exclusive licence to Springer Nature Limited 2020

\begin{abstract}
Background Obesity is an epidemic in New York City, the global epicenter of the coronavirus pandemic. Previous studies suggest that obesity is a possible risk factor for adverse outcomes in COVID-19.

Objective To elucidate the association between obesity and COVID-19 outcomes.

Design Retrospective cohort study of COVID-19 hospitalized patients tested between March 10 and April $13,2020$.

Setting SUNY Downstate Health Sciences University, a COVID-only hospital in New York.

Participants In total, 684 patients were tested for COVID-19 and 504 were analyzed. Patients were categorized into three groups by BMI: normal (BMI 18.50-24.99), overweight (BMI 25.00-29.99), and obese (BMI $\geq 30.00$ ).

Measurements Primary outcome was 30-day in-hospital mortality, and secondary outcomes were intubation, acute kidney injury (AKI), acute respiratory distress syndrome (ARDS), and acute cardiac injury (ACI).

Results There were 139 patients (27\%) with normal BMI, 150 patients who were overweight (30\%), and 215 patients with obesity (43\%). After controlling for age, gender, diabetes, hypertension, and qSOFA score, there was a significantly increased risk of mortality in the overweight (RR 1.4, 95\% CI 1.1-1.9) and obese groups (RR 1.3, 95\% CI 1.0-1.7) compared with those with normal BMI. Similarly, there was a significantly increased relative risk for intubation in the overweight (RR 2.0, 95\% CI 1.2-3.3) and obese groups (RR 2.4, 95\% CI 1.5-4.0) compared with those with normal BMI. Obesity did not affect rates of AKI, ACI, or ARDS. Furthermore, obesity appears to significantly increase the risk of mortality in males (RR 1.4, 95\% CI 1.0-2.0, $P=0.03$ ), but not in females (RR 1.2, 95\% CI 0.77-1.9, $P=0.40$ ).

Conclusion This study reveals that patients with overweight and obesity who have COVID-19 are at increased risk for mortality and intubation compared to those with normal BMI. These findings support the hypothesis that obesity is a risk factor for COVID-19 complications and should be a consideration in management of COVID-19.
\end{abstract}

These authors contributed equally: Mohamed Nakeshbandi, Rohan Maini

Supplementary information The online version of this article (https:// doi.org/10.1038/s41366-020-0648-x) contains supplementary material, which is available to authorized users.

Rohan Maini

Rohan.Maini@downstate.edu

1 Department of Medicine, Division of Infectious Disease, State University of New York, Downstate Health Sciences University, Brooklyn, NY, USA

2 Department of Emergency Medicine, State University of New York, Downstate Health Sciences University, Brooklyn, NY, USA

\section{Introduction}

The first case of coronavirus disease 2019 (COVID-19) emerged from Wuhan, China in December 2019. This new strain of coronavirus quickly spread globally, and COVID19 was classified as a pandemic by the World Health Organization in March 2020. The United States (U.S.), and

3 Department of Medicine, State University of New York, Downstate Health Sciences University, Brooklyn, NY, USA

4 Department of Epidemiology and Biostatistics, State University of New York, Downstate Health Sciences University, Brooklyn, NY, USA

5 Department of Bariatric Surgery, State University of New York, Downstate Health Sciences University, Brooklyn, NY, USA 
in particular New York City (NYC), eventually became the epicenter of severe acute respiratory syndrome coronavirus 2 [1].

Chronic conditions are associated with adverse outcomes in COVID-19. One such factor may be obesity [1], which has reached epidemic proportions in the U.S. and exacts a significant toll on the American healthcare system. In 2017, the Centers for Disease Control and Prevention reported that $42.4 \%$ of the American population was diagnosed with obesity [2]. The prevalence of obesity is particularly high in certain populations, such as non-Hispanic blacks and Hispanics [2]. These groups have also been reported to be disproportionately affected by the COVID-19 pandemic [3].

Previous studies have implicated obesity as a risk factor in severe COVID-19 outcomes, such as invasive mechanical ventilation [4], severe pneumonia [5], and increased hospitalizations [6]. Various mechanisms may be involved, including restrictive lung disease, lipo-toxicity, and induction of a proinflammatory state [7]. However, there is limited information describing the association between obesity and mortality in COVID-19, particularly in vulnerable racial and ethnic minority populations. The purpose of this study is to elucidate the association between obesity and COVID19 outcomes in a designated COVID-19-only center in East Flatbush, a community in Brooklyn, New York whose population is estimated to be $88 \%$ black and $34 \%$ obese [8].

\section{Methods}

\section{Study design}

This is a retrospective cohort study of patients tested for COVID-19 at SUNY Downstate Health Sciences University in New York, designated by Governor Andrew Cuomo as a COVID-only hospital. Testing was conducted via nasopharyngeal swab and subsequent reverse-transcription polymerase chain reaction (RT-PCR). The Cepheid machine was used for automation of RT-PCR. Data were collected and deidentified for all hospitalized patients through the Healthbridge Electronic Medical Health Record system. The Downstate institutional review board approved this study for publication.

\section{Subjects}

This study cohort had 684 patients who were tested for COVID-19 and 504 patients were ultimately used for analysis. The population included patients 18 years of age or older who were admitted from March 10th to April 13th 2020. Patients were excluded from the study if their COVID-19 test was negative; if body mass index (BMI) was not recorded in the electronic medical record or if the patient was underweight (defined as a BMI $<18.50 \mathrm{~kg} / \mathrm{m}^{2}$ ); and if they were still admitted to the hospital at the end of the study period. See Fig. 1 for exclusion criteria breakdown.

\section{Primary predictors}

Obesity was defined by BMI, calculated as weight (kilograms)/height ${ }^{2}$ (meters). Patients were categorized into three groups: normal (BMI 18.50-24.99 kg/m²), overweight (BMI $25.00-29.99 \mathrm{~kg} / \mathrm{m}^{2}$ ), and obese $\left(\right.$ BMI $\geq 30.00 \mathrm{~kg} / \mathrm{m}^{2}$ ). As mentioned above, underweight patients $\quad(\mathrm{BMI}<$ $18.50 \mathrm{~kg} / \mathrm{m}^{2}$ ) were excluded.

\section{Covariates}

Covariates were age, sex, diabetes mellitus, hypertension, and the quick sequential organ failure assessment (qSOFA) score measured on patient admission. Data were self-
Fig. 1 Study population criteria. Cohort was divided into three groups based off BMI (Body Mass Index).

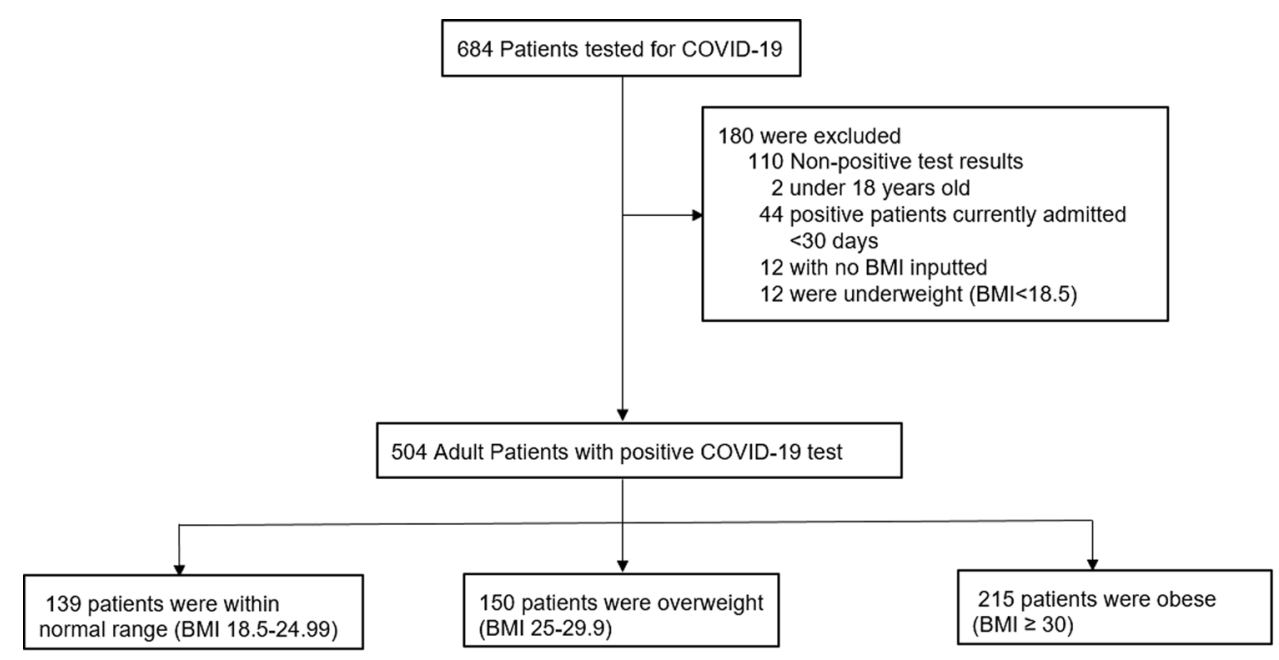


reported by the patient and added to the electronic record by the health care provider. qSOFA was defined as one point for each of the following criteria: altered mental status with Glasgow Coma Scale $<15$, tachypnea with respiratory rate greater than or equal to 22 , and hypotension with systolic blood pressure less than or equal to 100. qSOFA scores were dichotomized at a cutoff of 2 since patients with scores of 2 or greater have a higher risk of mortality [9].

\section{Outcomes}

The primary outcome was 30 day in-hospital mortality, while the secondary outcomes included intubation status, acute kidney injury (AKI), Acute Respiratory Distress Syndrome (ARDS), and acute cardiac injury (ACI). Acute kidney injury was diagnosed using the kDIGO criteria [10], while ARDS was defined by the Berlin criteria [11]. Acute cardiac injury was defined as elevation of troponin levels above the $99 \%$ of the upper reference limit occurring within 30 days of COVID-19 diagnosis.

\section{Statistical analysis}

Differences in demographics and clinical characteristics were compared across the three BMI groups, using $\chi^{2}$ or Fisher's exact tests for categorical variables and Kruskal-Wallis for continuous variables. Characteristics statistically significant at the 0.10 alpha level in bivariate analyses were included into the multivariable regression model [12]. Log-binomial regression models were used to determine whether any of the BMI groups was associated with an increase in the relative risk of outcomes upon adjustment for covariates. All statistical analysis was performed using SAS Studio 3.8 software.

\section{Results}

Table 1 shows the baseline characteristics of the study population. The mean age of the cohort was 68 , with 316 (63\%) patients greater than 65 years old. In this cohort, 263 $(52 \%)$ were male and 454 (90\%) were black. Baseline comorbidities included diabetes in $269(53 \%)$ and hypertension in 416 patients (83\%). There were 139 patients (27\%) with normal BMI, 150 patients with overweight (30\%), and 215 patients with obesity (43\%). The mean age of the group with obesity was 63 , while the normal range and groups with overweight were 70 and 71 , respectively.

After controlling for all covariates, there was a significantly increased risk of mortality in the patients with overweight (RR 1.4, 95\% CI 1.1-1.9, $P=0.003$ ) and patients with obesity (RR 1.3, 95\% CI 1.0-1.7, $P=0.04$ ) compared with those with normal BMI (Table 2). Similarly, there was significantly increased relative risk for intubation in the patients with overweight (RR 2.0, 95\% CI 1.2-3.3, $P$ $=0.01$ ) and patients with obesity (RR $2.4,95 \%$ CI $1.5-4.0$, $P=0.001)$ compared with the normal group (Table 3 ). Obesity did not demonstrate an association with AKI, ACI, or ARDS.

Subgroup analyses were also performed based on sex and age. A significantly increased risk of mortality was found in the group with obesity (RR 1.4, 95\% CI 1.0-2.0, $P=0.03$ ) compared with the normal BMI group in male patients, but not in female patients (RR 1.2, 95\% CI $0.77-1.9, P=0.40$ ) (See Appendix Table 1). Similarly, there was significantly increased relative risk for intubation in the group with obesity (RR $2.5,95 \%$ CI $1.4-4, P=$ 0.003) compared with the normal BMI group in male patients, but not in female patients (RR $2.395 \%$, CI $0.93-5.9, P=0.07$ ) (See Appendix Table 2). Age-stratified analyses demonstrate that among those 65 or older, being overweight significantly impacts mortality (RR $1.5,95 \%$ CI $1.2-2.0, P=0.002$ ) (See Appendix Table 3). In addition, among 45-64 year olds and those 65 years or older, obesity significantly impacts intubation (RR 3.0, 95\% CI 1.1-8.0, $P=0.03$ and RR $2.1,95 \%$ CI $1.1-3.8, P=0.02$ ), respectively (See Appendix Table 4).

\section{Discussion}

This study revealed that obesity is independently associated with adverse outcomes in COVID-19 patients when controlled for age, gender, hypertension, diabetes, and qSOFA score. Both groups with an elevated BMI showed an increased probability for mortality and intubation compared to the patients with normal BMI.

To our knowledge, this is the first large study to show the increased risk for mortality in this patient subset. The mechanism of this association is unclear but may be related to lipo-toxicity and the proinflammatory state induced by obesity. The excess free fatty acids induce lipo-toxicity, causing damage to organs such as the liver and pancreas. This leads to impairment of insulin receptors, contributing to insulin resistance, hypertriglyceridemia, and the progression to metabolic syndrome [13]. Furthermore, obesity creates a proinflammatory state, with increased expression of cytokines such as tumor necrosis factor alpha and interleukin-6, as well as acute phase reactants like Creactive protein (CRP) and amyloid antigen [13]. These cytokines cause damage to vascular endothelium, accelerating hypertension via renin-angiotensin-aldosterone system activation, atherosclerosis, and even thrombosis. Obesity has also been implicated in impairment of the adaptive immune response against influenza and other viruses [7]. 
Table 1 Characteristics of the study population at baseline according to BMI category $(n=$ 504).

\begin{tabular}{|c|c|c|c|c|c|}
\hline Characteristic & Total $n=504$ & $\begin{array}{l}\text { Normal } n= \\
139(27 \%)\end{array}$ & $\begin{array}{l}\text { Overweight } n= \\
150(30 \%)\end{array}$ & $\begin{array}{l}\text { Obese } n= \\
215(43 \%)\end{array}$ & $p$ value \\
\hline Age - mean \pm sd & $68 \pm 15$ & $70 \pm 16$ & $71 \pm 13$ & $63 \pm 14$ & $<0.001$ \\
\hline$<45 \mathrm{yrs}$ & $33(7 \%)$ & $9(6 \%)$ & $7(5 \%)$ & $17(8 \%)$ & $<0.001$ \\
\hline $45-64$ yrs & $155(31 \%)$ & $34(24 \%)$ & $28(19 \%)$ & $93(43 \%)$ & \\
\hline$\geq 65$ & $316(63 \%)$ & $96(69 \%)$ & $115(77 \%)$ & $105(49 \%)$ & \\
\hline Male sex $-n(\%)$ & $263(52 \%)$ & $84(60 \%)$ & $84(56 \%)$ & $95(44 \%)$ & 0.006 \\
\hline Race & & & & & 0.64 \\
\hline Black & $454(90 \%)$ & $126(91 \%)$ & $132(88 \%)$ & $196(91 \%)$ & \\
\hline White & $20(4 \%)$ & $8(6 \%)$ & $7(5 \%)$ & $5(2 \%)$ & \\
\hline Asian & $2(0.4 \%)$ & $1(1 \%)$ & 0 & $1(0.5 \%)$ & \\
\hline Hispanic & $3(0.6 \%)$ & 0 & $1(1 \%)$ & $2(1 \%)$ & \\
\hline Unknown & $25(5 \%)$ & $4(3 \%)$ & $10(7 \%)$ & $11(5 \%)$ & \\
\hline \multicolumn{6}{|l|}{ Comorbidities } \\
\hline Diabetes & $269(53 \%)$ & $72(52 \%)$ & $85(57 \%)$ & $112(52 \%)$ & 0.63 \\
\hline Hypertension & $416(83 \%)$ & $102(73 \%)$ & $127(85 \%)$ & $187(87 \%)$ & 0.003 \\
\hline Hyperlipidemia & $174(35 \%)$ & $38(27 \%)$ & $55(37 \%)$ & $81(38 \%)$ & 0.11 \\
\hline $\begin{array}{l}\text { Coronary Artery } \\
\text { Disease }\end{array}$ & $96(19 \%)$ & $30(22 \%)$ & $39(26 \%)$ & $27(13 \%)$ & 0.003 \\
\hline COPD & $41(8 \%)$ & $7(5 \%)$ & $9(6 \%)$ & $25(12 \%)$ & 0.05 \\
\hline Asthma & $41(8 \%)$ & $4(3 \%)$ & $12(8 \%)$ & $25(12 \%)$ & 0.009 \\
\hline $\begin{array}{l}\text { Chronic kidney } \\
\text { disease }\end{array}$ & $81(16 \%)$ & $21(15 \%)$ & $28(19 \%)$ & $32(15 \%)$ & 0.59 \\
\hline ESRD $^{\mathrm{a}}$ & $65(13 \%)$ & $18(13 \%)$ & $24(16 \%)$ & $23(11 \%)$ & 0.34 \\
\hline $\mathrm{qSOFA} \geq 2^{\mathrm{b}}$ & $156(31 \%)$ & $51(33 \%)$ & $48(32 \%)$ & $57(37 \%)$ & 0.12 \\
\hline $\begin{array}{l}\text { Current or } \\
\text { Former Smoker }\end{array}$ & $71(14 \%)$ & $16(12 \%)$ & $28(19 \%)$ & $27(13 \%)$ & 0.06 \\
\hline \multicolumn{6}{|l|}{ Outcomes } \\
\hline Intubation & $113(22 \%)$ & $17(12 \%)$ & $36(24 \%)$ & $60(28 \%)$ & 0.002 \\
\hline Acute kidney injury & $95(19 \%)$ & $31(22 \%)$ & $31(21 \%)$ & $33(15 \%)$ & 0.21 \\
\hline Acute cardiac injury & $160(32 \%)$ & $40(29 \%)$ & $49(33 \%)$ & $71(33 \%)$ & 0.66 \\
\hline $\mathrm{ARDS}^{\mathrm{c}}$ & $91(18 \%)$ & $18(13 \%)$ & $28(19 \%)$ & $45(21 \%)$ & 0.16 \\
\hline Mortality & $219(43 \%)$ & $51(37 \%)$ & $81(54 \%)$ & $87(40 \%)$ & 0.006 \\
\hline
\end{tabular}

${ }^{\mathrm{a}}$ End stage renal disease.

${ }^{b}$ Quick sequential organ failure assessment.

${ }^{\mathrm{c}}$ Acute respiratory distress syndrome.
Table 2 Adjusted relative risks for association between BMI category and mortality.

\begin{tabular}{lcc}
\hline Predictor & Relative risk $(95 \% \mathrm{CI})$ & $p$ value \\
\hline Overweight & $1.4(1.1-1.9)$ & 0.003 \\
Obese & $1.3(1.0-1.7)$ & 0.04 \\
Age & - & - \\
$\quad 45-64$ yrs & $2.7(1.0-6.9)$ & 0.04 \\
$\quad \geq 65$ & $4.5(1.8-11)$ & 0.002 \\
Male & $1.3(1.0-1.5)$ & 0.03 \\
Diabetes & $1.1(0.92-1.3)$ & 0.31 \\
Hypertension & $0.81(0.66-1.0)$ & 0.06 \\
qSOFA $\geq 2$ & $1.2(0.96-1.4)$ & 0.12 \\
\hline
\end{tabular}

Table 3 Adjusted relative risks for association between BMI category and intubation.

\begin{tabular}{lcc}
\hline Predictor & Relative risk $(95 \% \mathrm{CI})$ & $p$ value \\
\hline Overweight & $2.0(1.2-3.3)$ & 0.0 \\
Obese & $2.4(1.5-4.0)$ & $<0.001$ \\
Age & - & - \\
$\quad 45-64$ yrs & $2.1(0.81-5.7)$ & 0.12 \\
$\quad \geq 65$ & $2.0(0.77-5.3)$ & 0.15 \\
Male & $1.3(0.95-1.8)$ & 0.10 \\
Diabetes & $1.2(0.87-1.7)$ & 0.24 \\
Hypertension & $0.76(0.48-1.2)$ & 0.23 \\
qSOFA $\geq 2$ & $0.86(0.59-1.3)$ & 0.43 \\
\hline
\end{tabular}


This study's findings are consistent with previous studies that demonstrate an association between obesity and invasive mechanical ventilation [4]. This may be related to excess adipose tissue in the upper respiratory tract that results in obstruction of the airway and quicker progression to hypoxia. Patients with obesity also have increased intraabdominal pressure and a restrictive chest wall, resulting in decreased forced expiratory volume and forced vital capacity as well as decreased diaphragm contractility [7]. This contributes to lung dysfunction and subsequent desaturations leading to intubation.

Covariate analysis showed both age and the male gender to be significant independent predictors of mortality when controlling for other confounders. The stratified analysis showed that younger patients with obesity between 45-64 years of age have a relative risk of 3 for intubation compared with younger patients with normal BMI, while patients ages 65 or older have a comparably lower relative risk of 2.1. These results support the idea that obesity is raising the risk for COVID-19 complications in younger populations. Previous studies support this hypothesis, as it has already been shown that patients with obesity under the age of 60 are more likely to get admitted to acute and critical care settings [14].

Stratification by sex reveals worse outcomes in males over females, with higher risks of both mortality and intubation in males with obesity. This discrepancy may be related to anatomic variation: males tend to have more visceral fat, while females have more subcutaneous fat. Visceral fat has been shown to induce an inflammatory state with elevated CRP levels, while studies have shown that subcutaneous adipose tissue could be protective and lowers risk of mortality [15].

Demographic data reveals that most of the patients in this study reside in East Flatbush (See Appendix Fig. 1), a neighborhood with a higher rate of obesity (34\%) compare with Brooklyn overall (27\%) and NYC (24\%) (8). Factors that may contribute to this include lower income and limited access to higher determinants of health and healthy lifestyle options. For example, in East Flatbush, $80 \%$ of adults report eating one serving of fruits or vegetables per day (compared with $87 \%$ in NYC), and $32 \%$ report drinking one or more 12-ounce sugary drinks per day (vs $23 \%$ in NYC) [8]. The East Flatbush neighborhood also has a low density of supermarkets compared with bodegas, which have less healthy food options [8]. Of note, the prevalence of obesity in the admitted patients in our study (43\%) is higher than the average in the East Flatbush community (34\%). This may support the hypothesis that obesity increases rate of hospital admissions in COVID-19 [6].

This study has some limitations. Due to the observational nature of the study design, the potential for unmeasured residual confounding exists. Rates of all-cause mortality and intubation may be influenced by many other factors, such as socioeconomic disparities, advance directives and healthcare decisions, access to health insurance, dependence on public transportation, and overcrowding. Also, our study population is predominantly African American, a group with higher reported rates of obesity and other comorbidities [16], and may not be representative of the general U.S. population.

Despite these limitations, this study also has notable strengths: it is among the first to show an association between mortality and obesity, and uses relative risks as opposed to odds ratio, which is often computed in other studies and can exaggerate the risk ratio. Future research should address other risk factors and comorbidities that make COVID-19 patients high-risk, further clarifying healthcare decisions for the physician. We believe that this study supports the hypothesis that obesity is a risk factor for COVID-19 complications and should be considered by healthcare providers to dictate prevention and management of this virus.

Acknowledgements We would like to thank SUNY Downstate Health Sciences University for supporting the publication of this manuscript.

\section{Compliance with ethical standards}

Conflict of interest The authors declare that they have no conflict of interest.

Publisher's note Springer Nature remains neutral with regard to jurisdictional claims in published maps and institutional affiliations.

\section{References}

1. Goyal P, Choi J, Pinheiro L, Schenck E, Chen R, Jabri A, et al. Clinical Characteristics of Covid-19 in New York City. N Engl J Med. 2020. https://doi.org/10.1056/NEJMc2010419.

2. Centers for Disease Control and Prevention. Adult Obesity Facts I Overweight \& Obesity. https://www.cdc.gov/obesity/data/adult. html. Accessed 6 May 2020.

3. Dyer O. Covid-19: Black people and other minorities are hardest hit in US. BMJ. 2020;369:m1483. https://doi.org/10.1136/bmj.m1483.

4. Simonnet A, Chetboun M, Poissy J, Raverdy V, Noulette J, Duhamel A, et al. High prevalence of obesity in severe acute respiratory syndrome coronavirus-2 (SARS-CoV-2) requiring invasive mechanical ventilation. Obesity. 2020. https://doi.org/10. 1002/oby.22831.

5. Qingxian C, Fengjuan C, Wang T, Fang L, Liu X, Wu Q, et al. Obesity and COVID-19 Severity in a Designated Hospital in Shenzhen, China. SSRN Electron J. 2020. https://doi.org/10.2139/ssrn.3556658.

6. Petrilli C, Jones S, Yang J, Rajagopalan H, O'Donnell L, Chernyak $\mathrm{Y}$, et al. Factors associated with hospitalization and critical illness among 4,103 patients with. medRxiv. 2020:1-25. https:// doi.org/10.1101/2020.04.08.20057794.

7. Sattar N, McInnes I, Mcmurray J. Obesity a risk factor for severe COVID-19 infection: multiple potential mechanisms running title: Sattar et al.; Mechanisms for COVID Severity in Obesity. https:// doi.org/10.1161/CIRCULATIONAHA.120.047659.

8. NYC Department of Health and Mental Hygiene. Community Health Profiles - East Flatbush. New York City: NYC Health website; 2018. 
9. Jiang J, Yang J, Mei J, Jin Y, Lu Y. Head to head comparison of qSOFA and SIRS criteria in predicting the mortality of infected patients in the emergency department: a meta-analysis. Scand J Trauma Resusc Emergency Med. 2018;26:56. https://doi.org/10. 1186/s13049-018-0527-9.

10. KDIGO Clinical Practice Guideline for Acute Kidney Injury. https://doi.org/10.1038/kisup.2012.1.

11. ARDS Definition Task Force, Ranieri VM, Rubenfeld GD, Thompson BT, Ferguson ND, Caldwell E, Fan E, et al. Acute respiratory distress syndrome:the Berlin Definition. JAMA. 2012;307:2526-33. https://doi.org/10.1001/jama.2012.5669.

12. Vittinghoff E, Glidden DV, Shiboski SC, McCulloch CE. Regression methods in biostatistics: linear, logistic, survival, and repeated measures models. New York: Springer; 2015.
13. Redinger RN. The pathophysiology of obesity and its clinical manifestations. Gastroenterol Hepatol. 2007;3:856-63.

14. Lighter J, Phillips M, Hochman S, Sterling S, Johnson D, Francois $\mathrm{F}$, et al. Obesity in patients younger than 60 years is a risk factor for Covid-19 hospital admission. Clin Infect Dis. 2020;ciaa415. https://doi.org/10.1093/cid/ciaa415.

15. Tsao YC, Chen JY, Yeh WC, Li WC. Gender and age specific associations between visceral obesity and renal function impairment. Obes Facts. 2019;12:67-77. https://doi.org/10.1159/ 000496626.

16. Carnethon MR, Pu J, Howard G, Albert MA, Anderson C, Bertoni $\mathrm{AG}$, et al. Cardiovascular health in African Americans: a scientific statement From the American Heart Association. Circulation. 2017;136:e393-e423. 